\title{
EVALUATION OF CAMERA POSITIONS AND GROUND POINTS QUALITY IN A GNSS-NRTK BASED UAV SURVEY: PRELIMINARY RESULTS FROM A PRACTICAL TEST IN MORPHOLOGICAL VERY COMPLEX AREAS
}

\author{
E. Tufarolo ${ }^{1 *}$, C. Vanneschi $^{2}$, M. Casella ${ }^{3}$, R. Salvini ${ }^{1}$ \\ ${ }^{1}$ Department of Environment, Earth and Physical Sciences and Centre of Geotechnologies, University of Siena, Via Vetri Vecchi 34, \\ 52027 San Giovanni Valdarno, Italy \\ emanuele.tufarolo@unisi.it, riccardo.salvini@unisi.it \\ ${ }^{2}$ CGT Spinoff s.r.l., Strada Provinciale delle Miniere, Polo Industriale di Bomba, 52022 Cavriglia, Italy - vanneschi@cgt-spinoff.it \\ ${ }^{3}$ AeroDron s.r.1., Via Cremonese 35/A, 43126 Parma, Italy - m.casella@aerodron.com
}

KEY WORDS:UAV, Network Real Time Kinematic, Photogrammetry, Structure-from-Motion, Open Pit Mine, Positional accuracy assessment.

\begin{abstract}
Open pit mines localized in high mountains are probably one of the most complex environments for Structure-From-Motion (SfM) based photogrammetry. The case study presented in this paper refers to the realization of a detailed topographic mapping in the Torano marble basin (Apuan Alps, Italy) which needed, after decades of excavation activity, a new topographic survey.

Given the requested very high resolution, the time constraints and safety-related problems, a photogrammetric approach by a fixedwing Unmanned Aerial Vehicle (UAV) was chosen to carry out thesurvey of the basin. In addition, given the morphological complexity of the area, characterized by extreme steep slopes more than hundreds of meters high, and the necessity to minimize the fieldwork without sacrificing the work quality, an UAV equipped with a L1/L2 Network Real Time Kinematic (NRTK) Global Navigation Satellite System (GNSS) was used.

The scope of this work is to compare the accuracy of UAV derived 3D photogrammetric models realized with different approaches: by using traditional Ground Control Points (GCPs), by using the on-board Network Real Time Kinematic system for camera position detection, and a mix of both. At the end, we tested the quality of the models to verify the reachable levels of accuracy.
\end{abstract}

\section{INTRODUCION}

This paper analyses the accuracy of a bundle-adjustment photogrammetric process which uses photos from fixed-wing UAV flights over a morphological very complex mountain area. The work is part of a bigger project aimed to the creation of an updated topographic map, at a scale of 1:1,000, for an area more than 200 ha wide in the Torano marble basin (Carrara, Italy). The new topographic map was created from: a) the extraction of contour lines from the dense 3D point cloud, b) the editing of contour lines within a 3D GIS environment, c) the drawing in $3 \mathrm{D}$ of the remaining topographic features basing on the interpretation of the orthophotomosaic. Both, the dense point cloud and the orthophotomosaic, were generated by processing the digital images acquired by UAV flights and their bundleadjustment (topic of this work).

The Torano basin is characterized by high morphological complexity (Figure 1), consequence of the geology and the long history of the mining site attributable to the Roman Empire age. As base for the new topography, the dense point cloud and the high resolution orthophotomosaic were used as described in papers from Mancini et al. (2013), Gonçalves and Henriques (2015) and Westoby at al. (2012). Data was generated starting from digital images acquired by using a GNSS-NRTK equipped UAV. The work here presented focuses on the acquisition phase, the alignment of frames and their orientation through bundle-adjustment and SfM algorithms (Fonstad et al., 2013, Snavely et al., 2008; Ullman, 1979).

The analysis of the final model spatial accuracy was carried out by using different approaches.

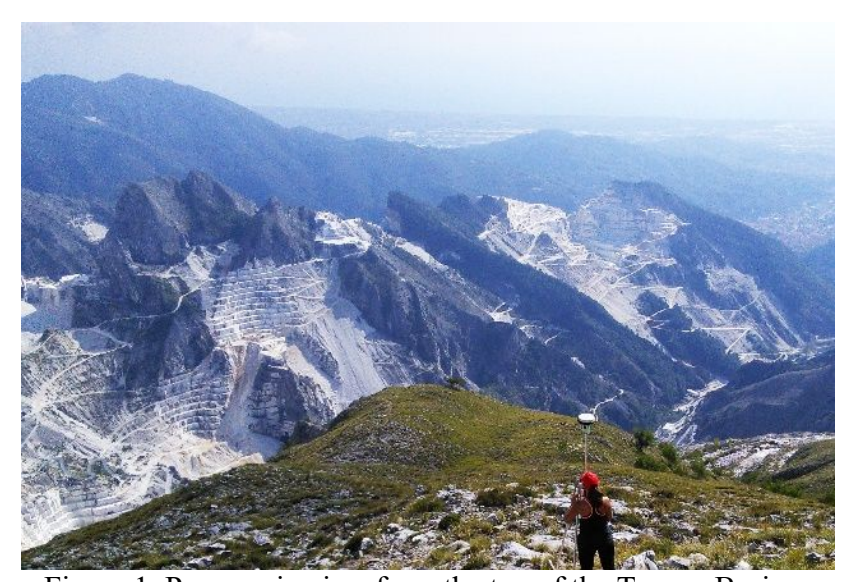

Figure 1. Panoramic view from the top of the Torano Basin; from the highest point to the main access road, the altitude difference is about $1,000 \mathrm{~m}$.

\subsection{Geographical and geological overview}

The Torano basin is located in the Apuan Alps (Tuscany, Italy), the most important marble extraction area of Europe with a production of about 1 million tons/years (Salvini et al., 2014).

The study area is located in the north-western part of the Carrara syncline, a pluri-kilometric isoclinal fold formed during the Tertiary orogenesis under greenschist conditions (Carmignani and Kligfield, 1990; Molli, 2008; Molli and Meccheri, 2012). 


\section{INSTRUMENTS}

\subsection{UAV}

The UAV used for the survey is an eBee plus RTK fixed-wing vehicle, produced by SenseFly (Parrot Group, Figure 2). The drone is made of foam, carbon and other composite parts with a nominal take-off weight of $1.1 \mathrm{~kg}$. The UAV can flight between 40 and $110 \mathrm{~km} / \mathrm{h}$ and can resist to a $12 \mathrm{~m} / \mathrm{s}$ wind. Three cells on board LiPo battery allow a maximum flight time of about 59 minutes. The remote control of the UAV is composed by a ground modem operating at $2.4 \mathrm{Ghz}$ allowing communications between UAV and Windows based PC that operates as ground station through the eMotion 3 software. The software is also used as bridge between UAV and real-time GNSS network through the mobile internet access. For this project the NETGEO Network VRS was used (Topcon Positioning Italy s.r.l.).

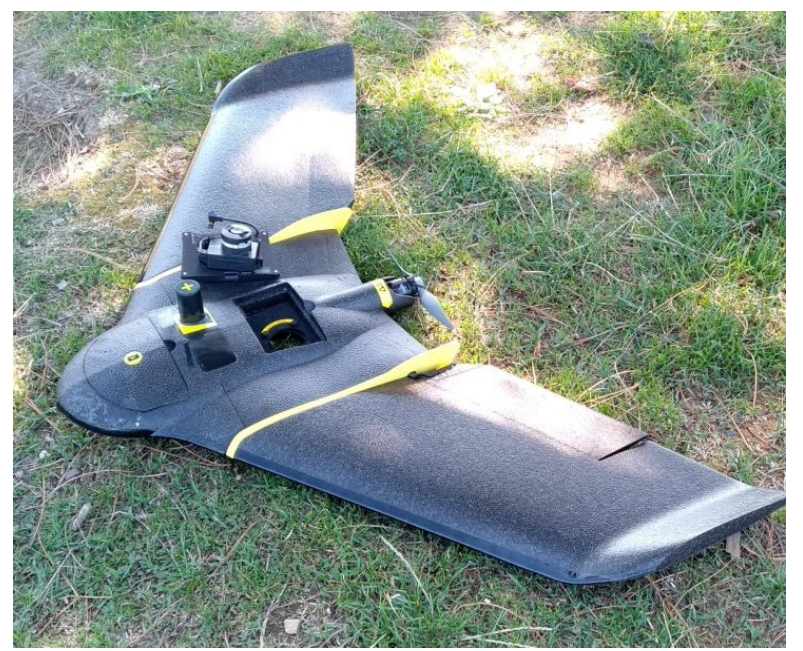

Figure 2. The eBee plus RTK fixed-wing UAV used in this work.

\subsection{Imaging sensor}

A S.O.D.A. sensor was installed on the eBee payload bay. S.O.D.A. is the acronym of Sensor Optimised for Drone Applications, a photogrammetric sensor specifically developed for small-commercial UAV operations. It is based on 1-inch 20megapixel RGB global shutter sensor (Table 1).

\begin{tabular}{|l|c|}
\hline Sensor & $1 "$ \\
Lens & $10.6 \mathrm{~mm} \mathrm{f} / 2.8$ \\
Resolution & $5472 \times 3648$ \\
Pixel size & $2.4 \mu \mathrm{m}$ \\
Ration & $3: 2$ \\
Image format & JPEG, DNG \\
\hline
\end{tabular}

Table 1. Main specifications of the used S.O.D.A. camera.

Due to the unavailability of the sensor's calibration certificate and with the aim of eliminating a possible variable in the process of accuracy evaluation, the interior orientation of the S.O.D.A. was initially estimated with the camera selfcalibration method (Fraser, 1997; Zhang, 2000; Remondino et al, 2006; Agisoft, 2008).

The calibration parameters (Table 2), calculated thanks to a reliable ground control points spatial distribution, were saved and utilised in the processing stage described in Paragraph 3.3.
Despite this, we have noticed that, even not fixing the camera calibration data, the bundle-adjustment step shows an extreme limited variation of the interior orientation parameters.

\begin{tabular}{|l|l|l|}
\hline Parameter & Value & Error \\
\hline $\boldsymbol{F}$ & 4399.57 & 0.027 \\
C $\boldsymbol{x}$ & 41.3461 & 0.021 \\
$\mathbf{C} \boldsymbol{y}$ & 19.1871 & 0.019 \\
B1 & 1.77081 & 0.0062 \\
$\mathbf{B 2}$ & -0.159396 & 0.006 \\
K1 & 0.0620122 & $6.5 \mathrm{e}-005$ \\
K2 & -0.420381 & 0.00046 \\
K3 & 0.929244 & 0.0013 \\
K4 & -0.60415 & 0.0012 \\
P1 & 0.00293547 & $1.8 \mathrm{e}-006$ \\
P2 & 0.00077971 & $1.5 \mathrm{e}-006$ \\
\hline
\end{tabular}

Table 2. Interior orientation parameters of the used S.O.D.A. sensor as calculated through the self-calibration method.

Figure 3 shows the distortion plot, based on self-calibration parameters, representing the image of residuals for the used S.O.D.A. sensor.

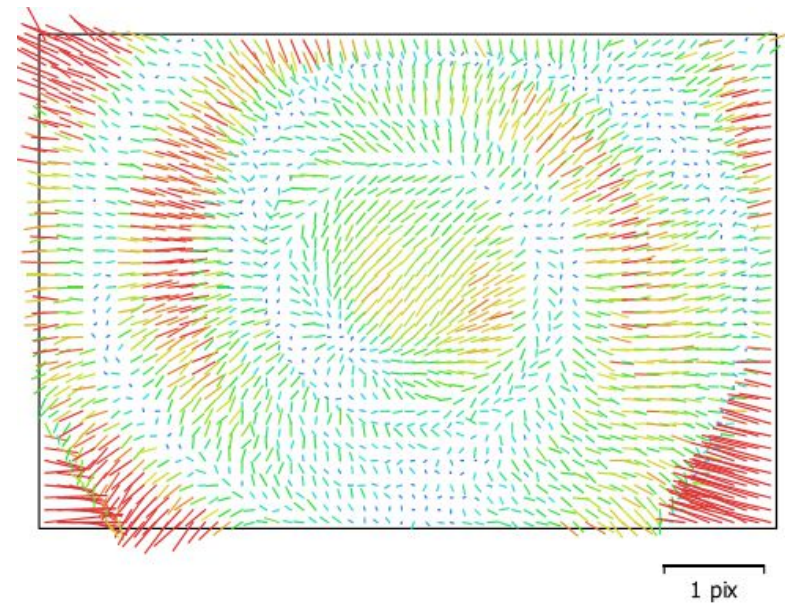

Figure 3. Image of residuals for the used S.O.D.A. sensor.

\subsection{Onboard GNSS}

The onboard GNSS based on a double-frequency GPS/GLONASS receiver operating at $20 \mathrm{~Hz}$, which acquires the NRTK data through the standard transmission protocol RTCM 3.x.

The internet mobile signal was always good during the survey but, in order to avoid signal disconnection (due to complex morphologies or temporary interferences), a GNSS reference station (Leica GS15 system) was used during the flight. The reference station allowed the post-processing of UAV RINEX data for camera positions acquired during possible NRTK down-times.

\section{DATA ACQUISITION AND PROCESSING}

\subsection{Flight plan and ground points distribution}

Due to the morphological complexity of the area, the UAV flight plans were heavily conditioned by the elevation differences within the basin, and could not follow a traditional planar approach; the relevant vertical differences between 
relatively close points on the ground required the use of a detailed and updated Digital Elevation Model (DEM) for the planning stage. The use of the DEM, alongside the NRTK approach, allowed the maintaining of a constant elevation of the UAV above ground level during the acquisition and a better security for the UAV itself. The adopted DEM is represented by a grid format ( $1 \mathrm{~m}$ pixel size) derived from an airborne aerial LiDAR survey (Light Detection And Ranging) supported by Tuscany Region in October 2017.

Figure 4 shows the planned Ground Points (GPs) distribution. Particular attention was given to the areas were the reconstruction algorithm might have more problems to returns high quality data (e.g. limits of the investigation, excavation area, high slopes).

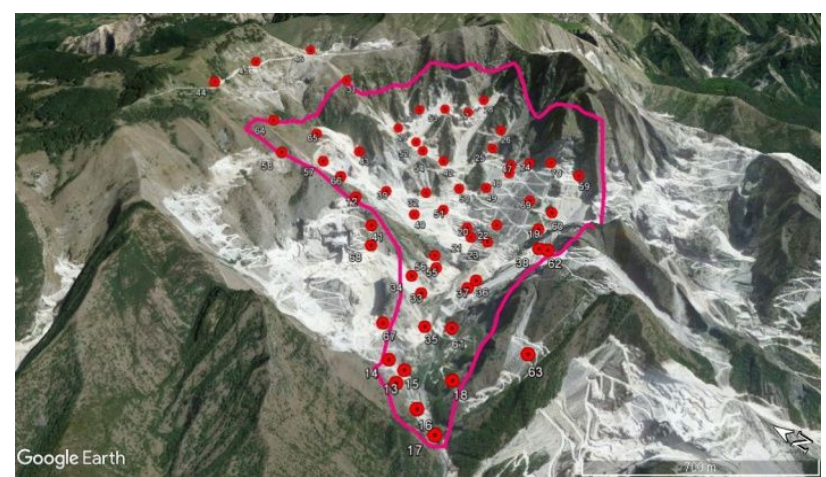

Figure 4. GP distribution (red dots) and limits of the investigation area in a Google ${ }^{\mathrm{TM}}$ Earth panoramic view.

Figure 5 and Figure 6 show a general view of the different camera positions during flights that, inevitably, follow the extremely complex morphology.

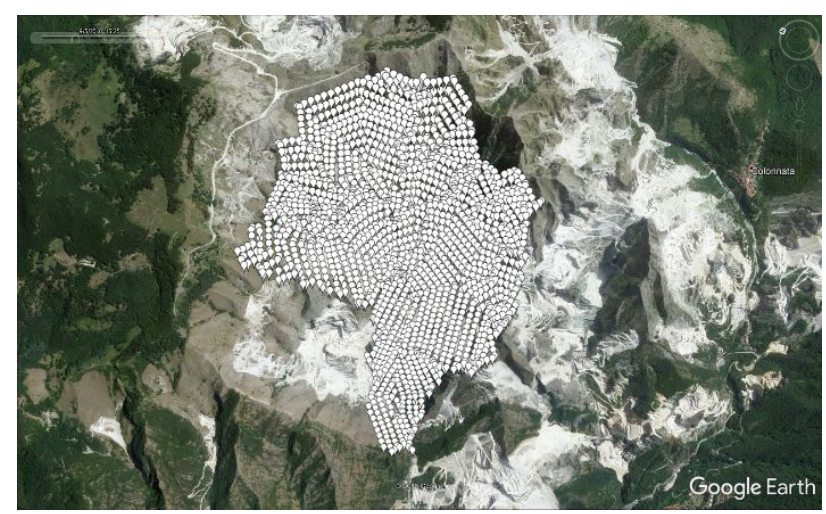

Figure 5. Spatial distribution of UAV camera positions (white icons) in a Google ${ }^{\mathrm{TM}}$ Earth nadiral view.

\subsection{Topographic survey}

Several artificial GPs were measured in the area of interest with particular attention posed on more complex areas and external limits. Three types of topographic points were acquired:

a) $75 \times 75 \mathrm{~cm}$ painted ground targets;

b) $50 \times 50 \mathrm{~cm}$ polyvinyl chloride (PVC) yellow/black targets;

c) man-made targets (e.g. corner of containers, edge of buildings).

The topographic survey of GPs was carried out by using two Leica GS15 GNSS receivers with NRTK correction from HxGN SmartNet (using the nearest Pieve Fosciana reference station). In case of lack of the mobile internet connection, the real-time correction was ensured by a couple of Leica 1200 reference stations, properly mounted in the higher zones of the basin. The communication was guaranteed by a direct radio connection or, if troubles occurred, by measuring the targets in the RapidStatic mode with acquisition times of about 15 minutes. In this latter, data was post-processed in laboratory by using Leica LGO 8.4 suite and contemporary satellite info recorded by the same reference station.

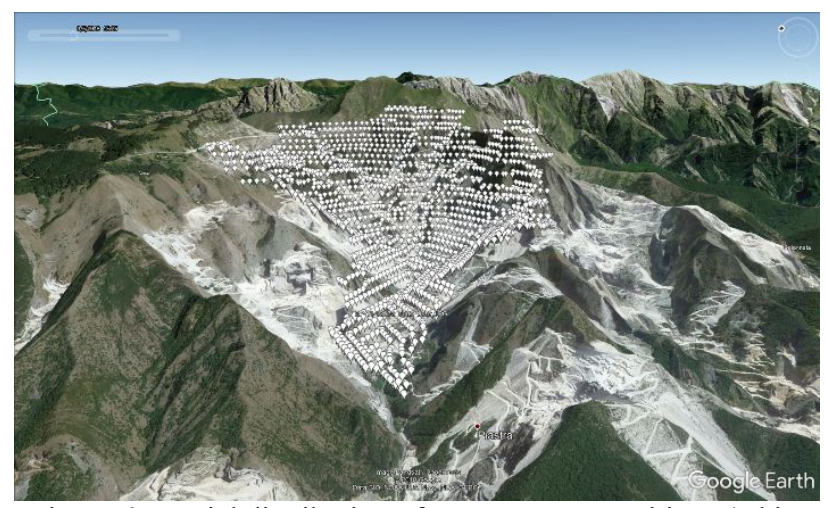

Figure 6. Spatial distribution of UAV camera positions (white icons) in a Google ${ }^{\mathrm{TM}}$ Earth pseudo-3D view.

\subsection{Image photogrammetric processing}

Survey data were pre-processed through eMotion 3 software in order to synchronize the frames and the coordinates of camera positions.

Coordinates of camera positions were principally obtained from a NRTK correction, but some frames, acquired during few radio-link losses, required a differential correction between onboard GNSS and the Leica GS15 local base station placed in an elevated close area. Also in these cases, data from the local GNSS base station were post-processed using the same reference station. Hence, all the coordinates of camera positions were characterised by high accuracy and were stored in exif metadata format, ready to be processed with the standard SfM workflow in Agisoft Photoscan Pro 1.4 code.

The total number of aligned camera stations was 2071 for a coverage area of $2.11 \mathrm{~km}^{2}$. The mean ground sampling distance is equal to $4.49 \mathrm{~cm}$. Image matching and alignment, at medium resolution using camera positions as reference for images preselection, was completed in 2 hours.

The coordinates of GPs acquired during the topographic survey (for a total of 64) were placed on the model as GCPs and Check Points (CPs) and used to optimise the exterior orientation through the bundle-adjustment. Both targets and camera position coordinates were projected in the Italian reference system Rete Dinamica Nazionale 2008/Zona 32 (RDN2008/TM32); altimetry was converted from ellipsoidal height to orthometric height by adopting info from ConveRgo software.

\section{ANALYSISOF RESIDUALS}

The photogrammetric 3D model was generated using different approaches in order to obtain, as much as possible, information relative to coordinates accuracy and frame alignment quality. In detail, the followed bundle-adjustments approaches were the following:

a) All the measured topographic points (ground points, GPs) were used as CPs and the bundle-adjustment was based only on camera position coordinates acquired thanks to the onboard NRTK system; 
b) A small number of measured topographic points was used as GCPs and the remaining as CPs. The bundle-adjustment was based on camera position coordinates and GCPs;

c) Topographic points were used as in the case of a traditional not-NRTK UAV survey; the bundle-adjustment was based on camera position coordinates and GCPs;

d) Topographic points were used as in the case of a traditional not-NRTK UAV survey; the bundle-adjustment was based only on GCPs.

Tables 3 and 4 report the Root Mean Square Errors (RMSEs) of camera positions, GCPs and CPs in the different configurations (i.e. a, b, c and d).

\begin{tabular}{|c|c|c|c|c|c|c|}
\hline Conf. & $\begin{array}{c}\text { Nr. of } \\
\text { used } \\
\text { GCPs }\end{array}$ & $\begin{array}{c}\text { Nr. of } \\
\text { used } \\
\text { CPs }\end{array}$ & $\begin{array}{c}\text { Use of } \\
\text { camera } \\
\text { positions }\end{array}$ & $\begin{array}{c}\text { GCP } \\
\text { XY } \\
\text { RMSE } \\
\text { (cm) }\end{array}$ & $\begin{array}{c}\text { CP XY } \\
\text { RMSE } \\
\text { (cm) }\end{array}$ & $\begin{array}{c}\text { Cam. } \\
\text { XY } \\
\text { RMSE } \\
\text { (cm) }\end{array}$ \\
\hline a & 0 & 64 & Yes & - & 83 & 11.6 \\
b & 14 & 50 & Yes & 12 & 42 & 18.8 \\
b & 24 & 40 & Yes & 7.7 & 30.48 & 22.15 \\
b & 35 & 29 & Yes & 6.31 & 28.62 & 23.17 \\
c & 49 & 15 & Yes & 6.06 & 15.58 & 25.26 \\
d & 14 & 50 & No & 0.5 & 7.51 & 89.2 \\
d & 24 & 40 & No & 0.6 & 7.08 & 89.8 \\
d & 35 & 29 & No & 0.92 & 5.43 & 86.4 \\
d & 49 & 15 & No & 1.18 & 4 & 86 \\
\hline
\end{tabular}

Table 3. Planimetric XY RMSEs $(\mathrm{cm})$ in different bundleadjustment configurations.

\begin{tabular}{|c|c|c|c|c|c|c|}
\hline Conf. & $\begin{array}{c}\text { Nr. of } \\
\text { used } \\
\text { GCPs }\end{array}$ & $\begin{array}{c}\text { Nr. of } \\
\text { used } \\
\text { CPs }\end{array}$ & $\begin{array}{c}\text { Use of } \\
\text { camera } \\
\text { positions }\end{array}$ & $\begin{array}{c}\text { GCP Z } \\
\text { RMSE } \\
\text { (cm) }\end{array}$ & $\begin{array}{c}\text { CP Z } \\
\text { RMSE } \\
\text { (cm) }\end{array}$ & $\begin{array}{c}\text { Cam. Z } \\
\text { RMSE } \\
\text { (cm) }\end{array}$ \\
\hline a & 0 & 64 & Yes & - & 188 & 16.82 \\
b & 14 & 50 & Yes & 64 & 152 & 22.27 \\
b & 24 & 40 & Yes & 46.63 & 92.91 & 31.85 \\
b & 35 & 29 & Yes & 37.97 & 84.88 & 34.64 \\
c & 49 & 15 & Yes & 43.11 & 85.4 & 38.67 \\
d & 14 & 50 & No & 1.95 & 12.52 & 176.2 \\
d & 24 & 40 & No & 3.42 & 8.99 & 175 \\
d & 35 & 29 & No & 2.83 & 9.35 & 177.4 \\
d & 49 & 15 & No & 2 & 5.4 & 177 \\
\hline
\end{tabular}

Table 4. Altimetric Z RMSEs $(\mathrm{cm})$ in different bundleadjustment configurations.

\section{DISCUSSION AND CONCLUSIONS}

UAV NRTK system, together with LiDAR derived DEM, resulted very useful for navigation purposes and allowed performing complex flight plans, necessary for obtaining the expected accurate outputs.

The high accuracy camera positions stored in the exif metadata format, allowed completing the alignment phase of the SfM process (in reference preselection modality) of 2071 images in 2 hours with a Dual Xeon Workstation, 128 Gigabyte RAM and 2 GPU.

Table 3 and 4 show the calculated results in different bundleadjustment configurations: the accuracy level was analysed on the CPs which were not used in bundle block adjustment but only as assessment points. Indeed, the relevant information for this work are the differences between the coordinates measured from the obtained block model and the real coordinates measured in the field trough the GNSS topographic survey. In the first approach (a) the resultant XY RMSE was equal to 83 $\mathrm{cm}$, while the RMSE on the $\mathrm{Z}$ coordinate was $188 \mathrm{~cm}$. The use of a certain number of GCPs (approach "b", 14 GCPs and 50 CPs) results in a lower error, with a planimetric RMSE of $42 \mathrm{~cm}$ and an RMSE of $152 \mathrm{~cm}$ on the $\mathrm{Z}$ coordinate. Passing to 49 GCPs and 15 CPs (approach "c") the calculated RMSE on XY coordinates was equal to $15.58 \mathrm{~cm}$, but on $\mathrm{Z}$ the RMSE remained still high $(85.4 \mathrm{~cm})$. The exclusion of camera coordinates from the bundle adjustment (approach "d") resulted in a better accuracy with a planimetric RMSE of $4 \mathrm{~cm}$ and a RMSE of $5.4 \mathrm{~cm}$ on the $\mathrm{Z}$ coordinate (i.e. with $49 \mathrm{GCPs}$ and 15 CPs configuration).

Anyway, with reference to the approach "d", the RMSE on CPs resulted quite low even with a small number of GCPs (only 14 well distributed points); in particular, the RMSEs resulted of $7.51 \mathrm{~cm}$ on $\mathrm{XY}$ and 12.51 on $\mathrm{Z}$ coordinate.

These errors, related to the CPs residuals, can be considered acceptable for the creation of a 3D model to be used for cartographic aims at a scale of 1:1,000. The errors in elevation, which result from models generated following the approach "a" (lower than $2 \mathrm{~m}$ ) are acceptable only for the production of $1: 10,000$ topographic maps.

To conclude, despite the technological progresses with SfM and NRTK on-board systems, the results of this research shows the necessity of measuring a homogeneous distribution of ground points in order to produce large-scale cartography. This is particularly true in a very complex morphological environment such as the mining area used for this test.

In addition, for a better understanding of the influence of camera position coordinates (recorded through a GNSS-NRTK receiver) on bundle block adjustment, a more detailed analysis may be beneficial. For example, a segmented approach, with a flight by flight analysis in subset areas could be useful to investigate the influence of the satellites signal degradation or the lack of real-time connection due to mobile internet signals instability, especially in the most depressed part of the site.

\section{ACKNOWLEDGEMENTS}

The authors would thank to Dumas F. and Omya GmbH personnel, especially Fantin G.P., Strauss H. and Pirkopf V., for the possibility given to carry out the project. Thanks also to Vernacchia L. and Rindinella A. for the help in the extensive fieldwork and Lanciano $C$. for the help in some image processing steps.

\section{REFERENCES}

Agisoft LLC, 2018. Agisoft Photoscan User Manual, Professional Edition, Version 1.4. agisoft.com

Carmignani, L., Kligfield, R., 1990. Crustal extension in the Northern Apennines: the transition from compression to extension in the Alpi Apuane core complex. Tectonics, 9, 12751303.

Fonstad, M.A., Dietrich, J.T., Courville, B.C. and Carbonneau, P.E., 2013. Topographic structure from motion: a new development in photogrammetric measurements. Earth Surface Processes and landforms, 20: 817-827.

Fraser, C. S., 1997. Digital camera self-calibration. ISPRS Journal of Photogrammetry and Remote sensing, 52(4), 149159.

Gonçalves, J. A. and R. Henriques, 2015. UAV photogrammetry for topographic monitoring of coastal areas. ISPRS Journal of Photogrammetry and Remote Sensing 104: 101-111. 
Mancini, F., Dubbini, M., Gattelli, M., Stecchi, F., Fabbri, S. and Gabbianelli G., 2013. Using unmanned aerial vehicles (UAV) for high-resolution reconstruction of topography: the structure from motion approach on coastal environments. Remote Sens., 5, pp. 6880-6898, 10.3390/rs5126880.

Molli, G., Meccheri, M., 2012. Structural inheritance and style of reactivation at mid-crustal levels: a case study from the Alpi Apuane (Tuscany, Italy). Tectonophysics 579, 74-87.

Molli, G., 2008. Northern Apennine-Corsica orogenic system: an updated review. In S. Siegesmund, B. Fügenschuh\& N. Froitzheim (Eds.), Tectonic aspects of the Alpine-DinarideCarpathian System (Vol. 298, pp.413-442). Geological Society of London, Special Publication.

Remondino, F., and Fraser, C., 2006. Digital camera calibration methods: considerations and comparisons. International Archives of Photogrammetry, Remote Sensing and Spatial Information Sciences, 36(5), 266-272.

Salvini, R., Giovannini, R., Vanneschi, C., Riccucci, S., Francioni, M. and Stead, D., 2014. The use of remote sensing for discontinuity mapping and analysis in a large marble quarry, Carrara, Italy. In International discrete fracture network engineering conference, Vancouver, Canada.

Snavely, N., Seitzs, N. and Szeliski R., 2008. Modeling the world from internet photo collections. International Journal of computer vision, 80: 189-210.

Ullman, S., 1979. The interpretation of visual motion. MIT Press, Cambridge, MA, USA.

Westoby, M.J., Brasington, J., Glasser N.F., Hambrey, M.J. and Reynolds, J.M., 2012. 'Structure-from-Motion' photogrammetry: a low-cost, effective tool for geoscience applications. Geomorphology, 179: 300-314

Zhang, Z., 2000. A flexible new technique for camera calibration. IEEE Transactions on pattern analysis and machine intelligence, 22. 